\title{
FRAGMENTO DE INSCRIPCIÓN DE COLONIA ROMVLA CON DEDICATORIA A UN JOVEN PATRONO
}

\section{FRAGMENT OF INSCRIPTION COLONIA ROMVLA WITH DEDICATION TO A YOUNG PATRON}

\author{
SALVADOR ORDÓÑEZ AGULLA*
}

Resumen: Se presenta la edición de una inscripción hallada en Sevilla que recoge la mención de un joven patrono. Palabras clave: Inscripción, Colonia Romula, patrono

\begin{abstract}
In this paper is presented the edition of an inscription mentioning a young patron found in Sevilla.

Key words: Inscription, Colonia Romula, patron
\end{abstract}

Lo que sí puede asegurarse es que no fue localizada in situ por los constructores del convento cuando edificaron sus cimientos en el siglo XV. Como demuestran las múltiples excavaciones realizadas en todo el entorno occidental del casco antiguo de Sevilla al oeste de la Alameda de Hércules, así como los análisis paleogeomorfológicos de ellas derivados (Barral 2009: 399 ss., 486 ss.), la zona en la que se incluye el Convento de Santa Clara no estaba ocupada en la antigüedad, encontrándose en plena llanura aluvial del Baetis y fuera del tell urbano que conformaba la terraza fluvial T13. Todo este entorno se encontraba sometido a una potente dinámica de inundaciones periódicas que se fue acentuando conforme el meandro del río se fue desplazando hacia el oeste desde sus posiciones de partida en la Alameda. Solo a partir del siglo XI, con el establecimiento del posible palacio abadí localizado bajo el actual Monasterio de San Clemente, o, con más seguridad, de la segunda mitad del siglo XIII, se documentará la presencia en el sector -incluso previamente al propio palacio de Don Fadrique- de niveles de ocupación que indican la definitiva antropización del entorno, sin por ello dejar de ser un área marginal de uso agrícola y vertedero, cuya completa urbanización tardará aún en completarse más de 200 años. 
La pieza que nos ocupa es un fragmento de pedestal de mármol blanco cremoso de grano grueso que conserva únicamente (59) $\mathrm{cm}$ en altura, una anchura máxima de $76 \mathrm{~cm}$ (pie); no se ha podido medir su grosor $^{2}$. Al pie, de arriba abajo, cuenta con sendas molduraciones, en cyma reversa inversa y en cyma recta inversa. Con estos datos, puede intuirse que lo que queda de ella es apenas una cuarta parte, o menos, de su tamaño original. El campo epigráfico tiene $29 \mathrm{~cm}$ de ancho y $(7-8,2) \mathrm{cm}$ de altura, y está delimitado por una moldura perimetral de $2,2 \mathrm{~cm}$; a su vez el campo se encuentra enmarcado por un friso corrido $(5,5 \mathrm{~cm}$ de anchura en las bandas verticales, $5 \mathrm{~cm}$ en la horizontal) con decoración vegetal de roleos acantiformes, con evidentes paralelos formales con los que presentan una serie de piezas procedentes del Suroeste hispano, y concretamente del Bajo Guadalquivir (Beltrán 1988; Beltrán 2007) ${ }^{3}$. En este caso los relieves de zarcillos acantiformes aparecen como gruesos tallos acompañados de las pertinentes rosetas, lo que ubicaría esta pieza hispalense, desde el punto de vista del diseño de los roleos, dentro del grupo B de Beltrán. Como se ha señalado para el conjunto anterior, es muy probable que la pieza que presentamos proceda también de un taller lapidario bético, a tenor del hecho de que ocho de los doce ejemplares con esta decoración emplean mármol procedente de las canteras de Almadén de la Plata, en la provincia de Sevilla.

Se conservan únicamente dos líneas del texto -de las 5 o 6 , al menos, de que constaría la inscripción-, que no presentan problemas de lectura. La paginación no está equilibrada, alineándose el texto por la derecha con un fuerte sangrado de la última línea. El campo epigráfico está pulido, aunque al día de hoy, como resultado de la restauración del lugar donde se halla embutida, presenta numerosas adherencias y concreciones debidas al revoco de la pared, que dificultan la nitidez de la reproducción fotográfica. El espacio interlineal es de $2 \mathrm{~cm}$, mientras que la distancia de la última línea de texto a la moldura inferior del campo epigráfico es de $1,7 \mathrm{~cm}$. Las letras son capitales librarias de grabado profundo, con refuerzos marcados, que miden 2,5 $\mathrm{cm}$; en la 1.1 no todas las letras se conservan completas. I longa en ambas íes de Iuneni, de 3,3 cm de altura. No se aprecia en autopsia la presencia de signos de interpunción ni restos de líneas de guía. Particularidades

2. Las medidas consignadas son las que hoy pueden tomarse tras la restauración del muro en que se encuentra embutida la pieza.

3. Algunas, como CIL II 1186 o CIL II 1172, procedentes de la misma Hispalis.

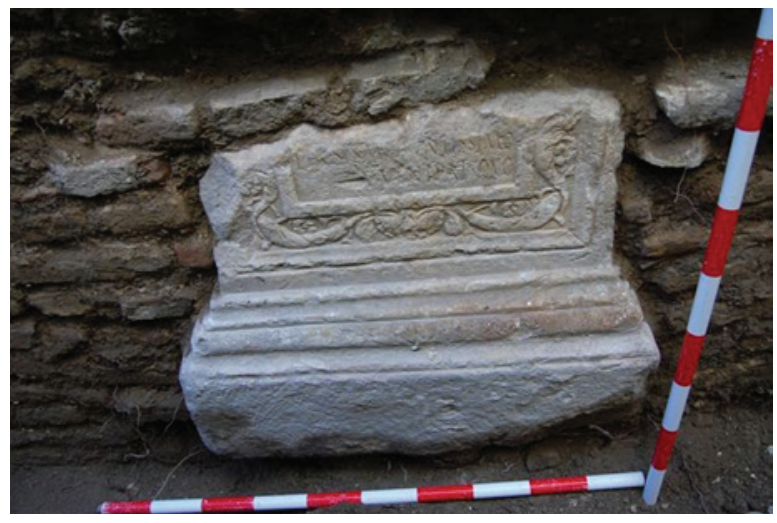

Figura 1.

paleográficas: $\mathrm{P}$ con óculo sin cerrar; travesaño de la $\mathrm{T}$ ligeramente ondulado y corto; óculo de $\mathrm{R}$ es ovalado; $\mathrm{O}$ también ovalada (Figuras 1 y 2$)^{4}$.

La lectura del texto es:

\section{PENSIS MERENTISSIMO IVVENI PATRONO}

\section{------ / [------] / pensis merentissimo / iuиeni patrono}

Cabe datar esta pieza en torno a fines del siglo II d.C., o inicios del III d.C., tanto por el tipo de letra utilizado como por el empleo del superlativo, cuyo uso se generaliza precisamente en estas fechas (Beltrán 1980: 342; Alföldy 1975: 474 ss.).

En el estado actual de la inscripción no puede establecerse qué tipo de patronato es el contemplado en el texto, que pudiera referirse bien a una relación de tipo personal, bien a un patronato de índole colegial, o a un patronato ciudadano ${ }^{5}$. Realmente la reconstrucción que se dé a la parte del texto inicialmente conservada, [---] pensis, condiciona las posibles interpretaciones del patronato que aquí se menciona. Pues si optamos por considerar que se oculta una origo $^{6}$, hay que pensar que

4. Fotos P. Oliva.

5. En relación con Hispalis, y de este último tipo, solo conocemos un personaje, probablemente un senador o un ecuestre, quizá el mismo prefecto de Egipto homónimo de 297 d.C. (PIR ${ }^{2}$ A 1048a), a través de una inscripción de Conobaria fechada en 297 d.C., vid. CILA II.3, $992=$ HEp 7, 780; Gimeno, Stylow 2007: 195 n 4. Un posible patronato sobre Hispalis por parte del senador L. Liuius Ocella (CIL X 6319), cuestor en Hispania entre 41 y 27 a.C., ha sido sugerido por Eck (1991: 150).

6. O incluso un domicilium bajo una forma adjetival, como en CIL XIII 6236 (domo Termestinus) o CIL II ${ }^{2}$ /7, 987 (domo Reginensis). No creemos que pueda tratarse de las últimas sílabas de un cognomen derivado de un topónimo, al modo del Vetina Italicensis de 


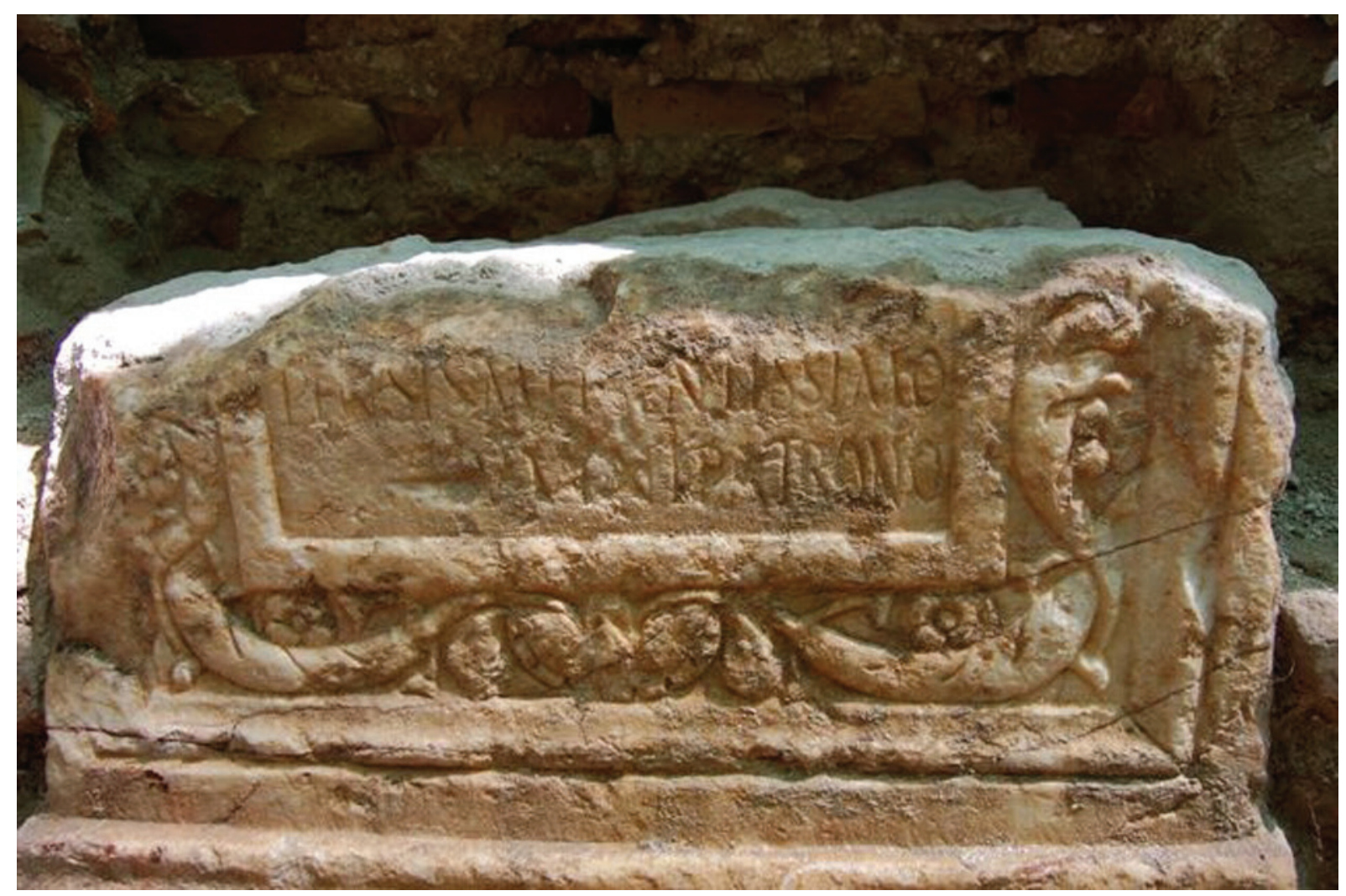

Figura 2.

entonces la inscripción recogería al inicio el nombre del homenajeado y quizá algún dato añadido relativo a su persona y carrera, a lo que seguiría el nombre del dedicante, consignándose al final la condición de iuuenis y patrono del primero. En ese caso estaríamos ante un vínculo de tipo privado y desigual en el que del dedicante, un liberto que llevaría la misma origo que su manumisor ${ }^{7}$, solo podríamos saber que era originario de alguna de las ciudades cuya origo se desarrolla en la forma [---]pensis, como Ilipa (Ilipensis), Irippo (Iripensis), Orippo (Orippensis), Basilipo (Basilippensis), Iulipa (Iulipensis), Serippo (Serippensis), Ripa (Ripensis) o Serpa (Serpensis). Naturalmente, no es posible discernir de cuál de ellas pudo provenir el personaje en cuestión, aunque la cercanía a Hispalis de las tres primeras abogaría en principio por cualquiera de ellas. La identificación de Ilipa con Alcalá del Río y de Orippo con la Torre de los Herberos, en Dos Hermanas

Dig. 48.19.27 pr. (PIR 809) o el L. Marinius Italicensis de CIL XII 1921; el repertorio de Kajanto (1965) no recoge ningún ejemplo de cognomen en -pensis.

7. Dig. 50.1 .27 (Ulp.).
(Sevilla), no presenta hoy ninguna $\mathrm{duda}^{8}$; por su parte, Irippo se ha asimilado recientemente con el potente asentamiento de la Mesa de Gandul, en Alcalá de Guadaíra (Pascual 2001; Pascual 2003). Basilippo se sitúa en la Torre del Cincho, Carmona (Sevilla), a 24 millas de Hispalis en la vía hacia Antikaria ${ }^{9}$. Iulipa es la actual Zalamea de la Serena, en la Baeturia Turdulorum, en el conuentus Cordubensis ${ }^{10}$. Ripa, por su parte, conocida solo a través de la referencia de Plinio (3.10) a los oppida del conuentus Cordubensis, se ha ubicado en la finca de San Sebastián, en las cercanías de Lopera (Jaén) (Stylow, Morena, Pérez 1997). Serpa se sitúa en la actual ciudad homónima del Alentejo ${ }^{11}$. Descartable nos parece, en fin, intentar vincular a este personaje con

8. Sobre Ilipa, vid. ahora el conjunto de trabajos en Ferrer et al. (2007); sobre Orippo, Fernández et al. (1980), Ponsich (1991: 109111); Escacena y Padilla (1992: 34-35); Fernández et al. (1997).

9. CIL II $1373=$ CILA II.3, $907=H E p$ 15, 313. Rodríguez (1979-80: 425-435)

10. $C I L \mathrm{II}^{2} / 7,904=H E p 5,122$, con mención de un [I] ulipensis. Sobre esta ciudad, Stylow (1991); $C I L \mathrm{II}^{2} / 7$, pg. 206; Saquete y Pavón (2009).

11. CIL II $971=H E p ~ 7,1152$. 
otras origines como Iappioppensis ${ }^{12}$, la ciudad de Philippi en Asia o la provincia de la Dacia Ripensis.

Otra opción que cabría entender tras la mención de [---]pensis podría ser la de una inscripción en la que patronus y cliens perteneciesen ambos a las elites ciudadanas, algo que, aunque con menos frecuencia, está suficientemente atestiguado en los usos sociales romanos recogidos en la práctica epigráfica; se trataría en este último caso de la acepción de patronus como 'protector' o 'benefactor'"13.

Tampoco es descartable la referencia a algún cargo, colectivo u organización política ciudadana, del tipo decuriones et plebs [---]pensis, o, más factible, ordo [---]pensis. En este último caso, y siempre en el supuesto de que la pieza no haya sido desplazada, estaríamos ante un fenómeno no muy frecuente, pero sí atestiguado epigráficamente, la dedicación de un homenaje a un personaje de una comunidad determinada por parte del ordo de otra. Así, el senado de colonia Iulia Genetiua costea los funerales y la sepultura de un chico de doce años que fallece y es enterrado en Corduba, y lo mismo hace en esta capital el ordo de colonia Augusta Firma con relación al joven decurión astigitano C. Furnius Fortunatus ${ }^{14}$; cabe recordar también en este sentido la dedicación elevada por los cinco ordines de Hispalis, Italica, Asido, Siarum y Callet a un personaje socialmente eminente, Lucia Auircia Aciliana, en esta última comunidad ${ }^{15}$, o los honores decretados al duunviro Iunius Auitus en el municipio Lunense por parte de los ordines de esta misma ciudad, de la de los dumienses y la de los polconenses ${ }^{16}$.

Al margen de estas conjeturas, otra posibilidad sería entender aquí una mención de las fórmulas suis impensis, ex impensis o similar, aunque en este caso éstas casi siempre van ligadas a un verbo al final del texto, que aquí no consta.

12. $H E p 12,561=A E 2002,758=A E 2004,770$. Referido a $F a$ malicao, un lugar de la Citerior junto a Conimbriga.

13. Sobre la vinculación de patronato de notables y aristócratas locales con miembros de los estamentos senatorial y ecuestre y altos cargos de la administración provincial, reflejada en la epigrafía africana, vid. Saller (1982: 10, 195-199); Saller (1989: 54 ss.).

14. $A E 1962,76=C I L \mathrm{II}^{2} / 7,307=H E p$ 5, 321; CIL II $\mathrm{II}^{2} / 7,306$. En Sevilla $C I L$ II $1192=C I L A$ II.1, $38=H E p ~ 4,809$ presenta el homenaje del ordo de un municipio (¿Ilipa?, ¿Inlipa?) a una mujer, pero no está claro que la inscripción proceda realmente de Hispalis.

15. ILS $6920=$ CILA II.4, 1220. En esta misma línea, CIL II 3251-3252, un ciudadano de Baesucci honrado por su propia comunidad y por Laminium, Tugia y Viuatia, o AE 1993, $909=$ HEp 5, 94, homenaje funerario conjunto de dos colonias, Emerita y Metellinum, a un Iluir de Metellinum que lo fue en tres ocasiones.

16. $C I L \mathrm{II}^{2} 5 / 218$.
La fórmula laudatoria merentissimus es poco empleada en la epigrafía latina. Ya S. G. Harrod había señalado que en la misma Vrbs es raro su uso (Harrod 1909: 1), y ciertamente solo una docena de testimonios pueden aducirse al respecto, una cifra muy poco relevante $^{17}$. En todo el imperio, según muestra una consulta a $E D C S$, no alcanzan las 150 menciones. El empleo de este término se hace de forma preferente en el círculo de las relaciones familiares, especialmente para referirse a las cualidades de madres cónyuges, maridos e hijos, y, con menor incidencia, de otros niveles de los vínculos familiares -frater, nurus, parentes, soror, auunculus - o de las relaciones de dependencia en el marco privado-seruus, conseruus, contubernalis, ancilla, alumna-, incluidos, obviamente, los patroni. No obstante, aunque poco frecuentes, existen testimonios de la aplicación del adjetivo merentissimus a patronos que se mueven en el ámbito público municipal ${ }^{18}$; tales son los casos, por ejemplo, del flamen provincial M. Iulius Serenianus Adoptiuus, a quien la Prouincia Hispania Citerior le eleva una estatua en Tarraco como patrono merentissimo ${ }^{19}$, o el del procurator ecuestre y gobernador C. Iulius Pacatianus, a quien la colonia Aelia Augusta Italica honra en Vienna, en la Narbonense, con la misma fórmula ${ }^{20}$.

Por otro lado, en su inmensa mayoría los testimonios epigráficos que incluyen este superlativo se elevan en contexto funerario, siendo excepcionales los que se usan en inscripciones honoríficas ${ }^{21}$; en este sentido resulta llamativo que entre ellos figuren varias inscripciones hispalenses, como la que a fines del siglo II o inicios del III recoge el homenaje a L. Horacio Víctor, dos veces IIuir y merentissimus ciuis al que el populus le eleva una estatua por su muy espléndida liberalidad para con su patria y pueblo 22 ; o la que el corpus [ole]

17. CIL VI 3611, 2584, 9533, 12253, 14322, 18449, 19083, 26036, 34612; AE 1975, 77, ICVR 7, 18488; ICVR 10 26349. Por el contrario, benemerens, y, en general, los derivados de mereor, son muy frecuentes, vid. Huttunen (1966); también en Hispania, donde se emplea especialmente entre patronos y libertos, vid. Curchin (1981: 180).

18. No se contempla este término en el catálogo de virtudes municipales de Forbis (1996)

19. $C I L I^{2} / 14.2 .1,1145=R I T 284=H E p 15,341=A E 2006,695$.

20. $C I L \mathrm{XII} 1856=I L S 1353=A E 1960,247=I L N \mathrm{~V} .1,65=A E$ 2007, 1631; vid. sobre él Pflaum (1960) nº 229.

21. En Hispania solo en un pequeño grupo, entre las cuales CIL II $2211=C I L$ II $^{2} / 7,332$ (Corduba); AE 1897, $100=$ RIT 284 (Tarraco); CIL II 5141 (Ossonoba) y CIL II 5812=AE 1946, 120 (Sasamón).

22. CIL II $1185=$ CILA II.1, $27=H E p$ 14, 348. El testimonio de J. Breval recoge la variante meritissimo, frente al resto de autoridades, vid. Canto (296 n 39 y $\left.345 n^{\text {a }} 150\right)$. En este caso, como en el de la inscripción que nos ocupa, se emplea la forma de merentissimus + 
ari[orum] splend[idissi]mum elevó a M. Iulius Hermesianus, difusor olei ad annonam Vrbis y curator corporis oleariorum ${ }^{23}$. En cualquier caso, dada la escasa porción del texto conservado en el epígrafe de Santa Clara, no puede establecerse con certidumbre si nuestra pieza es de carácter funerario u honorífico, si bien nos parece más factible esta última opción.

De la misma manera, tampoco es posible determinar con fiabilidad el sentido con el que se emplea aquí el término iuuenis, aunque probablemente no se trate de una referencia a un miembro de una organización de jóvenes -collegium iuиenum, iuuenes, iuuentus ${ }^{24}$. Un repaso a las menciones de esta voz en los listados epigráficos muestra que la mayor parte de las veces este término, cuando se consigna aislado y en singular, se usa, sin sentido técnico, para aludir a la juventud del personaje mencionado, usualmente con sus correspondientes calificativos en superlativo, como en este testimonio de Sevilla ${ }^{25}$. En cualquier caso, los testimonios epigráficos antiguos dejan también claro que por iuuenis podía entenderse la condición de edad de un individuo entre sus dieciséis y hasta alrededor de sus cincuenta años ${ }^{26}$, y que era un hecho habitual y admitido socialmente la tendencia a la introducción de niños, adolescentes y jóvenes en la vida pública y su presentación en sociedad como adultos completos, como la referencia al patronato deja entrever aquíi ${ }^{27}$.

\section{BIBLIOGRAFÍA}

ALFÖLDY, G. (1975): Die römischen Inschriften von Tarraco. Berlin, Walter de Gruyter.

BARRAL MUÑOZ, M. Á. (2009): Estudio geoarqueológico de la ciudad de Sevilla. Sevilla, Universidad de Sevilla.

sustantivo, frente al uso generalizado que constata la forma sustantivo + merentissimus.

23. HEp 10, $576=A E 2001,1186=A E 2003,129=A E 2003$, $130=A E 2007,755=H E p$ 17, 122. Pero $c f$. la lectura alternativa de Remesal (2008: 351: ob mer[ita eius]).

24. Vid. sobre ellas, Ginestet (1991).

25. Kleijwegt (1994: 80): “when somebody is styled iuvenis it does not necessarily mean that the individual had been a member of an offficial collegium", especialmente $\mathrm{n}^{\mathrm{a}} 9$, con un listado de testimonios en los que la mención del término iuuenis no permite establecer ningún vínculo expreso de la pertenencia del individuo a un collegium o una iuuentus.

26. Aul. Gell. NA 10.28; Kleijwegt (1991: 55, 103-104).

27. Sobre la ambigua posición del joven en la sociedad antigua, vid. especialmente Kleijwegt (1991: passim); Kleijwegt (1994).
BELTRÁN FORTES, J. (1988): "Frisos de roleos acantiformes en los monumentos epigráficos de la Bética", Baetica VIII: 163-194.

- (2007): "Soportes hispanorromanos del suroeste peninsular: pedestales y altares decorados con frisos vegetales", XII Congressus Internationalis Epigraphiae Graecae et Latinae. Provinciae Imperii Romani inscriptionibus descriptae. Barcelona, Institut d'Estudis Catalans: 123-132.

BELTRÁN LLORIS, F. (1980): Epigrafía latina de Saguntum y su territorium. Valencia, Diputación provincial de Valencia.

CANTO, A. (2004): "Los viajes del caballero inglés John Breval a España y Portugal: novedades arqueológicas y epigráficas a partir de 1726", RPA 7.2: $265-364$.

CURCHIN, L. (1981): "Familial epithets in the epigraphy of Roman Spain", Mélanges offerts en hommage au R. P. Étienne Gareau = Cahiers d'Etudes Anciennes XIV: 179-182.

ESCACENA CARRASCO, J. L. y PADILLA MONGE, A. (1992): El poblamiento romano en las márgenes del antiguo estuario del Guadalquivir. Écija, Gráficas Sol.

FERNÁNDEZ GÓMEZ, F. et al. (1980): "Excavaciones en Orippo 'Las Moriscas' (Dos Hermanas, Sevilla)", NAH XXVIII: 25-60.

FERNÁNDEZ GÓMEZ, F. et al. (1997): Orippo en la Antigüedad. Las excavaciones arqueológicas de 1979 a 1983. Dos Hermanas, Ayuntamiento de Dos Hermanas.

FERRER ALBELDA, E. et al. (eds.) (2007): Ilipa antiqua. De la prehistoria a la época romana. Alcalá del Río, Ayuntamiento de Alcalá del Río.

FORBIS, E. (1996): Municipal virtues in the Roman Empire. The evidence of Italian honorary inscriptions. Stuttgart, Teubner.

GIMENO PASCUAL, H. y STYLOW, A. U. (2007): "La epigrafía romana de Las Cabezas de San Juan", Arqueología en el Bajo Guadalquivir. Prehistoria y Antigüedad de Las Cabezas de San Juan. Sevilla, Universidad de Sevilla.

GINESTET, P. (1991): Les organisations de la jeunesse dans l'Occident Romain. Bruxelles, Latomus.

HARROD, G. S. (1909): Latin terms of endearment and of family relationship. A lexicographical study based on volume VI of the Corpus Inscriptionum Latinarum. Princeton, Princeton University.

HUTTUNEN, P. (1966): "Some notes on the use of the verb mereo (mereor) in republican political terminology and in pagan inscriptions", Arctos IV: 47-61. 
KAJANTO, I. (1965): The Latin cognomina. Michigan-London, Ann Arbor UMI.

KLEIJWEGT, M. (1991): Ancient Youth. The ambiguity of youth and the absence in Greco-Roman society. Amsterdam, J. C. Gieben.

KLEIJWEGT, M. (1994): "Iuvenes and Roman imperial society”, Acta classica 37 (1994) 79-102.

OLIVA MUÑOZ, P.; JIMÉNEZ SANCHO, A. y TABALES RODRÍGUEZ, M. Á. (2003): "Primera fase de estudios arqueológicos en el Real Monasterio de Santa Clara de Sevilla", AAA'2003 II: 336-351.

OLIVA MUÑOZ, P.; TABALES RODRÍGUEZ, M. Á. y MORA VICENTE, G. M. (2007): Real Monasterio de Santa Clara. Palacio y cenobio. Sevilla, Colegio de Aparejadores.

PASCUAL BAREA, J. (2001): “Irippo y la Mesa de Gandul (Alcalá de Guadaíra): 'la fortificación del río Ira’ en época turdetana”, Congreso Internacional Fortificaciones en el entorno del Bajo Guadalquivir: 169-177. Alcalá de Guadaíra, Ayuntamiento de Alcalá de Guadaíra.

- (2003): "La ciudad romana de la Mesa de Gandul como emplazamiento de Irippo y en relación a Lucurgentum y Alcalá de Guadaíra", Actas del III Congreso de Historia de Andalucía, Historia Antigua: 389-407. Córdoba, Cajasur.

PFLAUM, H.-G. (1960): Les carrières procuratoriennes équestres sous le Haut-Empire Romain. Paris, Paul Geuthner.
PONSICH, M. (1991): Implantation rurale Antique sur le Bas-Guadalquivir. Madrid, Casa de Velázquez.

REMESAL, J. (2008): “Olearii”, Epigrafia 2006. Atti della XIV Rencontre sur l'épigraphie in onore di Silvio Panciera con altri contributi di colleghi, allievi e collaboratori: 349-373. Roma, Quasar.

RODRÍGUEZ HIDALGO, J. M. (1979-80): “Anotaciones en torno a Basilippo. La Torre del Cincho", Habis 10-11: 425-435.

SALLER, R. P. (1982): Personal patronage under the early Empire. Cambridge, Cambridge University Press.

- (1989): "Patronage and friendship in early Imperial Rome: drawing the distinction", Patronage in Ancient Society: 49-62. London-New York, Routledge.

SAQUETE CHAMIZO, J. C. y PAVÓN TORREJÓN, P. (2009): "La vertebración territorial de la Baeturia Turdulorum en época imperial romana", Andalucía romana y visigoda: 151-165. Roma, L'Erma di Bretschneider.

SOLÍS GUZMÁN J. (coord.) (2006): Real Monasterio de Santa Clara. Historia y descripción. Sevilla, Colegio de Aparejadores.

STYLOW, A. U. (1991): "El municipium Flavium V(---) de Azuaga (Badajoz) y la municipalización de la Baeturia Turdulorum", StudHist 9: 11-28.

STYLOW, A.U.; MORENA LÓPEZ, J. A. y PÉREZ DAZA, F. (1997): "Notas para la localización de Ripa (Plinio, N. H. III, 10). A propósito de un nuevo hallazgo epigráfico", $A A C$ 8: 181-196. 